\title{
DIMENSIONES SOCIALES EN ÉTICA GLOBAL. NUEVAS PERSPECTIVAS EN EDUCACIÓN
}

\author{
Marta T. Fracapani Cuenca de Cuitiño ${ }^{1}$
}

Resumen: El artículo hace referencia a algunos hitos a considerar en los programas de educación superior, con el fin de adoptar un punto de vista amplio y crítico de la experiencia, el conocimiento y el aprendizaje, que incluya la búsqueda de la comprensión de las relaciones entre nuestras vidas y las personas a través del mundo. Refiere además una experiencia local en la provincia de Mendoza. A partir de la evaluación de la residencia de profesionales, se promueve la elaboración de una ley que la reglamenta y propicia profundos cambios, tales como la intersectorialidad, la organización y pertenencias a redes, y la prioridad en primer nivel en salud y la interdisciplina. Las residencias de los profesionales del equipo de salud, como recurso educativo para formar ciudadanos y profesionales críticos y preparados en un mundo globalizado, son parte del proceso transformador de salud, que pueden ayudar a priorizar este valor en todas las políticas. La bioética, como ética aplicada, aporta su método y fundamenta la justicia en el sistema.

Palabras clave: bioética, educación superior, globalización, formación profesional, residencias profesionales

\section{Social dimensions in global ethics. New perspectives in education}

Abstract: This paper refers to some landmarks to consider in university training programs, with the aim to adopt a wide point of view by reflecting on experience, knowledge and learning, including the search for comprehending the relations of our lives and of people around the world. Furthermore, it refers to local experience in the province of Mendoza, Argentina. The elaboration of a norm to rule professionals in residence is promoted based on their evaluation, favoring deep changes, such as actions between sectors, organization and net membership and the first level priority on health and inter discipline. The residences of health care professionals, as educational resource to train citizens and critical professionals prepared for a globalized world, is part of the transforming process in health care, which could help to give priority to this value in all policies. Bioethics, as applied ethics, provides its method and lays the foundation for justice in the system.

Key words: bioethics, university education, globalization, professional training, professional residences

\section{Dimensóes sociais em ética global. Novas perspectivas em educaçáo}

Resumo: $\mathrm{O}$ artigo faz referência a alguns ritos a serem considerados nos programas de educação superior, com a finalidade de adotar um ponto de vista amplo e crítico da experiência, do conhecimento e da aprendizagem, que incluía a busca da compreensão das relaçóes entre nossas vidas e as pessoas através do mundo. Refere, ademais, uma experiência local na província de Mendoza. A partir da avaliaçáo da residência de profissionais promove-se a elaboração de uma lei que a regulamenta e propicia profundos câmbios, tais como a intersetorialidade, a organização e o pertencimento a redes, a prioridade no primeiro nível em saúde e a interdisciplinaridade. As residências dos profissionais da equipe de saúde, como recurso educativo para formar cidadãos e profissionais críticos e preparados num mundo globalizado, são parte do processo transformador de saúde, que pode ajudar a priorizar este valor em todas as políticas. A bioética, como ética aplicada, aporta o seu método e fundamenta a justiça no sistema.

Palavras-chave: bioética, educação superior, globalização, formação profissional, residências profissionais

\footnotetext{
${ }^{1}$ Facultad de Ciencias Médicas, Universidad Nacional de Cuyo, Mendoza, Argentina

Correspondencia: mtfracapani@gmail.com
} 


\section{Introducción}

Los sistemas educativos de América Latina plantearon, desde su origen, la necesidad de socializar políticamente a los estudiantes. Las ideas que desarrolla Domingo Faustino Sarmiento sobre la escuela popular, o las que plantea Horace Mann, en "Massachussets", tienen un propósito esencialmente cívico: integrar grupos de diferente origen social y cultural, con el fin de construir nacionalidad y ciudadanía. También tenían el objetivo de crear un nuevo orden político que enfrentara el orden de los caudillos, los cuales, desde las guerras de la independencia, compiten por el poder en América Latina.

El desafío es pasar de esta visión tradicional de la educación a una visión ampliada. Para ello hay que valorar que se requiera promover el desarrollo de competencias (conocimientos, habilidades y actitudes) para saber, saber hacer, cómo se tiene que saber ser y, además, para participar y para convivir. Implica actuar en todos los niveles educativos, en diferentes ámbitos, con altos niveles de calidad, con contenidos explícitos en los programas de las diferentes áreas y sobre los ejes transversales del currículum, así como en el clima institucional, pedagógico y organizacional.

Desarrollar una perspectiva global implica adoptar un punto de vista amplio y crítico de la experiencia, el conocimiento y el aprendizaje, incluyendo la búsqueda de la comprensión de las relaciones entre nuestras propias vidas y de las personas a través del mundo. Para adoptar una perspectiva global necesitamos acentuar nuestra comprensión de las fuerzas sociales, políticas y ambientales que configuran nuestra existencia.

La academia siempre ha sido internacional, pero el desafío es comprender las complejidades y matices del contexto global, un mundo académico forjado con desigualdades en el que el mercado y las fuerzas comerciales dominan de manera creciente. Lo importante es asegurar que la globalización no devenga en el neocolonialismo del siglo $\mathrm{XXI}(1)$.

Jane Knight define "internalización” como el proceso de integración de una dimensión internacional, intercultural o global en los propósitos, funciones o implementación del currículo en los niveles institucionales o nacionales(2). La internalización no es un fin en sí mismo, sino un camino para mejorar la calidad de la educación, la investigación, etc. Si deliberamos y trabajamos en determinar los fines que hacen necesario este cambio en los sistemas educativos, y cómo debieran las instituciones educativas mejorar su capacidad para dar respuesta a los actuales y futuros desafíos sociales en distintos ámbitos, como por ejemplo el sanitario, la universidad estará cumpliendo con la responsabilidad social que le compete.

Haremos referencia a algunos hitos en los programas de educación superior $y$, finalmente, a una experiencia local, en la provincia de Mendoza, Argentina, de formación de posgrado de profesionales de la salud.

\section{Conferencia Mundial sobre Educación Supe- rior 2009}

La Conferencia Mundial sobre Educación Superior, se celebró en la sede de la UNESCO, en París. del 5 al 8 de julio de 2009. En ella se analizaron cambios desarrollados a la educación superior desde la primera Conferencia Mundial de 1998. Se centró en la nueva dinámica de la educación superior y la investigación para el cambio social y el desarrollo, señalando como una de las tareas prioritarias de la universidad el poner a disposición el conocimiento existente y generar conocimientos nuevos al servicio de la construcción social. También se debatió sobre costes, internalización, provisión transfronteriza y la importancia de la acreditación para la garantía de calidad y de los rankings.

Se señaló que el concepto de bien público en la educación superior está directamente relacionado con los roles que sus instituciones deben desempeñar en la sociedad. En ellas se forman los profesionales que alcanzarán las posiciones de mayor responsabilidad. Por lo tanto, la educación superior tiene una importante responsabilidad pública respecto de los contenidos curriculares, la ética y los valores que trasmite, y por tanto requiere una revisión a fondo de su misión.

Además, se coincidió en que era necesario armonizar el conocimiento económicamente per- 
tinente con el conocimiento humano y social pertinente. La educación superior debe ser eficaz y eficiente, pero a su vez requiere la pertinencia académica y social.

Ofreció una plataforma global para el debate y el análisis con visión de futuro de la educación superior y la investigación. Alcanzó acuerdos sobre recomendaciones orientadas a las acción, para responder mejor a las nuevas dinámicas de la agenda para el desarrollo de las políticas e instituciones de educación superior y la investigación, así como para hacer frente a los desafíos mundiales y construir economías basadas en el conocimiento que sean más integradoras, equitativas y sostenibles.

La conferencia se centró en tres temas principales:

- La función de la educación superior en el tratamiento de los grandes desafíos mundiales presentes y futuros, por ejemplo, seguridad alimentaria, cambio climático, gestión del agua, diálogo intercultural.

- Compromiso de la sociedad y responsabilidad social de la educación superior.

- Fomentar la excelencia para acelerar el desarrollo de África.

En las sesiones paralelas hubo tres subtemas:

- Internacionalización, regionalización y mundialización.

- Equidad, acceso y calidad.

- Aprendizaje, investigación e innovación.

Las nuevas dinámicas señaladas para la educación superior fueron:

- Demanda.

- Diversificación.

- Creación de redes.

- Aprendizaje a lo largo de toda la vida.

- Tecnologías de la información y la comunicación.
- Responsabilidad social.

- Evolución del papel de los gobiernos.

También se destacó como relevante en las nuevas dinámicas la expansión de las tasas de matrícula, las tecnologías de la información y comunicación (TICs) y la globalización.

El Grupo Latinoamericano, a través de sus ministerios de educación y jefes de delegaciones, logró que se plasmara en el comunicado final el concepto de educación superior como bien público, y no como servicio público, así como derecho humano fundamental, como se había acordado en la Conferencia Regional de Educación Superior, en octubre de 2008, en Cartagena.

Por lo expuesto, aunque la Conferencia Mundial de la UNESCO sobre educación superior no tenga una definición normativa para que sus conclusiones sean acotadas por los Estados miembros y sólo sean recomendaciones, representa un verdadero hito en el que más de mil delegados, de unos 150 países, han debatido y acordado cómo la educación superior debe contribuir a la erradicación de la pobreza y al desarrollo sostenible. Al vincular los criterios de calidad a la pertenencia, la equidad y el desarrollo sostenible, y no sólo a su eficiencia, se visualiza a la educación superior como un bien público social, un derecho humano y universal y un deber del Estado. Esta concepción genera nuevos roles profesionales y nuevas responsabilidades en todos los involucrados en las funciones institucionales. Por ejemplo, centrarse más en los aspectos interdisciplinarios y promover el pensamiento crítico y la ciudadanía activa, así como hacer realidad los derechos humanos.

\section{Consenso Global sobre la Responsabilidad So- cial de las Facultades de Medicina(3)}

Este documento propone a las facultades de medicina, en el siglo XXI, un nuevo paradigma de excelencia, tal como lo hiciera el Informe Flexner, para el siglo XX, en 1910. Los retos son distintos. En lugar de conseguir una formación médica más científica y efectiva, hoy se propone la mejora de la calidad, equidad, relevancia y efectividad en la provisión de los servicios asistenciales, reducción de los desajustes respecto de las prioridades socia- 
les, redefinición de los roles para los profesionales de la salud, así como demostración de su impacto sobre el estado de salud de la gente.

Con este fin, 135 organizaciones y líderes con responsabilidades en la educación de los profesionales, en su regulación, o de las políticas de salud, provenientes de todo el mundo, tomaron parte durante ocho meses en un proceso Delphi de tres ciclos, que culminó en una conferencia entre el 10 y 13 de octubre de 2010, para alcanzar un consenso. Así surgieron diez directivas estratégicas para las facultades de ciencias médicas, con el fin de devenir socialmente responsable y por lo tanto ser capaces de:

- Dar respuesta a las necesidades de salud, actuales y futuras, y a las demandas de la sociedad.

- Reorientar sus prioridades educativas, de investigación y de servicios para que provean dichas necesidades.

- Reforzar su gobernanza, así como su partenariazgo con otros agentes sociales interesados (stakeholders).

- Utilizar la evaluación y acreditación basada en su desempeño y en los resultados obtenidos.

El consenso recomienda reforzar sinergias entre organizaciones y redes existentes, para progresar de forma consensuada hacia una acción global, mediante una serie de actividades:

- La defensa y promoción del valor del consenso global.

- La consulta con el fin de adaptar e implementar el consenso global.

- La investigación para el diseño de los estándares que reflejan la responsabilidad social.

- La coordinación global para compartir experiencia y proporcionar apoyos.

El desafío es que las instituciones educativas demuestren su contribución a la mejora del desempeño de los sistemas de salud de la población, no sólo por la confección de programas educativos orientados hacia problemas prioritarios de salud, sino también por una mayor participación en la capacidad de anticiparse a las necesidades de salud y de recursos humanos de un país. Así como asegurar que los graduados tengan trabajo donde más se los necesita para proveer los servicios más necesarios

Se necesita:

- Nuevo paradigma de excelencia para las instituciones académicas.

- Nuevo conjunto de estándares y un mecanismo de acreditaciones con el fin de evaluar la capacidad para tener un mayor impacto en la salud.

Hay consenso en 10 áreas temáticas para lograr que una escuela de medicina mejore su capacidad para dar respuesta a los futuros desafíos sanitarios de la sociedad. Para incrementar esta capacidad se incluye el uso de la autoevaluación y la evaluación por pares, y se sugiere cómo debería evaluarse el avance hacia estos objetivos:

- Previsión de las necesidades de la salud de la sociedad.

- Asociación de las necesidades de salud y los grupos de intereses.

- Adaptación a los cambios en el rol de los médicos y otros profesionales de la salud.

- Fomento de la educación basada en resultados.

- Generar una gobernanza responsable de las facultades de medicina, para que sean capaces de responder.

- Redefinir el ámbito de incumbencias de los estándares educativos, de investigación y de prestación de servicios.

- Mejora continuada de la calidad de la educación, investigación y prestación de servicios.

- Establecer mecanismos de acreditación. 
- La responsabilidad social es un valor universal.

- Las sociedades locales serán las que aprecien los logros.

\section{Conferencia Sanitaria Panamericana. 64a Sesión del Comité Regional OPS-OMS 2012(4)}

Esta conferencia se realizó en Washington D.C. del 17 al 21 de septiembre de 2012. Punto 4.9 del orden del día provisional CSP28/14, Rev. 1 del 20 de septiembre de 2012.

El documento conceptual denominado "Bioética: Hacia la integración de la ética en el ámbito de la salud" constituye otro de los hitos que queremos destacar. Con este documento se muestra la importancia de integrar las consideraciones éticas en las políticas de salud, la atención médica y la investigación con seres humanos relacionada con la salud, y en el desarrollo y la adopción de nuevas tecnologías que tienen repercusión sobre la salud.

Anteriormente, en Panamá, en junio de 2007, se había presentado otro instrumento político que orientaba la elaboración de futuros planes nacionales de salud y los planes estratégicos de todas las organizaciones interesadas en la cooperación en materia de salud con los países de las Américas. Esta Agenda de Salud para las Américas (20082017) había resaltado la importancia de promover la bioética: "La bioética tiene que difundirse y aplicarse más en los países de las Américas, para resguardar la calidad de la investigación y el respeto a la dignidad de las personas, salvaguardar la diversidad cultural y la aplicación de los conocimientos en salud, así como su aplicación en la toma de decisiones en salud pública”(5).

Entre los antecedentes de la $28^{\text {a }}$ Conferencia Sanitaria Panamericana, se hace referencia a la fecunda acción desarrollada por el Programa Regional de Bioética de la OPS, que se estableció en 1993 en Santiago, Chile, con la misión de cooperar con los Estados Miembros de la Organización y sus entidades públicas y privadas en el desarrollo conceptual, normativo y aplicado de la bioética en sus relaciones con la salud. El Programa inició sus actividades en 1994, en el marco de un convenio entre la OPS, la Universidad de Chile y el gobierno chileno, generando una masa crítica de personas entrenadas en bioética, con énfasis en ética de la investigación.

En el punto 9 se señala algo que nos parece importante reproducir, porque ilumina caminos a seguir: "El Programa Regional de Bioética ha fortalecido principalmente el desarrollo académico de la disciplina. Persiste, sin embargo, una brecha entre los académicos formados en bioética y los responsables de la toma de decisiones y los profesionales de la salud que lidian directamente con los problemas que son objeto de la bioética. La incorporación de la bioética en la formulación de políticas públicas sanitarias todavía constituye un desafío pendiente en la Región. A pesar de contar con personas formadas en ética de la investigación, hay Estados Miembros que todavía no disponen de un marco normativo y regulatorio para la investigación con seres humanos, ni de pautas y mecanismos para abordar los problemas éticos que surgen en la atención de la salud".

También se afirma que no todas las acciones orientadas a la mejora de la salud son éticamente aceptables. Más aún, la actividad de salud pública no es éticamente neutra, sino que implica juicios de valor sobre lo correcto y lo justo. Para incorporar la ética en el ámbito de la salud hay que identificar y analizar los criterios y principios éticos en juego; estos pueden ser varios e incluso entrar en conflicto. No es posible suponer sencillamente que las distintas actividades y políticas que buscan mejorar la salud son aceptables desde el punto de vista ético sin antes haber realizado un análisis fundamentado en bioética. Tampoco es posible suponer que los marcos legales son suficientes para elucidar o resolver todos los conflictos éticos. La ley es fundamental para determinar los estándares mínimos que deben respetarse. Lo requerido por la ley es, sin embargo, solo una de las dimensiones del actuar ético; la ética con frecuencia dicta acciones que van más allá de lo que requiere la ley. De hecho, no es posible ni deseable que la ley cubra todo el espectro de la vida moral de individuos o sociedades.

La bioética debe incorporarse en el trabajo regular de profesionales de la salud y responsables de las decisiones de política, de modo que las políticas 
públicas sanitarias estén fundamentadas en principios bioéticos.

Con el fin de determinar si una política o intervención en salud pública es ética, es preciso evaluar si hay evidencia de que: a) la intervención es efectiva para lograr los objetivos propuestos; b) los beneficios de la intervención para la salud pública superan los dańos y cargas que pudieran resultar de la intervención; c) el objetivo de salud pública no puede alcanzarse de una manera distinta que implique menos cargas para la población; d) la intervención va a resultar en la menor cantidad posible de cargas o efectos adversos, y e) las cargas y los beneficios se van a distribuir de manera equitativa, minimizando si es posible injusticias sociales anteriores. Además, por el imperativo ético de tratar a las personas con respeto, deben utilizarse procedimientos justos, tales como una deliberación pública para determinar qué cargas son consideradas aceptables por la comunidad en cuestión.

Con el objetivo de incorporar la ética de manera sólida y sistemática en las distintas áreas de la salud, se propone una variedad de mecanismos, que incluyen: Fortalecimiento de las capacidades de bioética: enfatizando la aplicación del análisis bioético; priorizando a los responsables de las decisiones políticas y al personal de los organismos gubernamentales de salud; identificando y evaluando constantemente las capacidades existentes para enfocar las actividades de capacitación en las necesidades específicas de la Región y así proceder eficazmente; creando y fortaleciendo las redes nacionales y regionales para elaborar una agenda común que integre los distintos esfuerzos en marcha y así avanzar eficientemente.

Apoyo a los países mediante: la formulación y ejecución de políticas, planes, programas y regulaciones en las áreas propias de la bioética; la incorporación de la bioética en la formulación y ejecución de políticas, planes, programas y reglamentaciones en las distintas áreas de la salud, con el fin de garantizar que las políticas, planes, programas y reglamentaciones resultantes sean éticamente sólidos.
Experiencia local. Ley 7.857 de la Provincia de Mendoza de la República Argentina, de Residencias Profesionales(6)

En la provincia de Mendoza, República Argentina, se analizaron diferentes aspectos de la realidad local con el fin de planificar estrategias para mejorar el sistema de residencias. Se trabajó, entre otras, con las siguientes premisas:

- Hacen falta centros de salud y hospitales que brinden la atención sanitaria adecuada.

- Hay suficiente evidencia científica y social sobre que los problemas de salud de la población no se resuelven construyendo más hospitales y medicalizando a los ciudadanos(7).

- Si se quiere proteger la salud de los ciudadanos, que es un derecho humano fundamental, se debe desarrollar una estrategia que ponga a la salud en el centro de todas las políticas(8).

- La salud pública tiene un carácter multiprofesional. Para que esta multiprofesión pueda articularse adecuadamente, parece razonable solicitar que los graduados en distintas profesiones compartan una visión multidisciplinaria común que les permita abordar los determinantes sociales de la salud y tomar conciencia del impacto de las políticas no sanitarias.

- La puesta en marcha de la estrategia de salud en todas las políticas es hoy imprescindible.

- La capacitación de los profesionales de la salud es un valor ańadido para el sistema sanitario, para la economía, para la sociedad y para la calidad de vida de la población.

- Las Residencias en América Latina han pasado por distintas etapas históricas. Las mismas etapas se identifican en Argentina, aunque en nuestro país, a diferencia de los otros de la región, las Residencias se han mantenido ajenas a la universidad.

- Se visualiza una necesidad de cambio en las Residencias, ya que realidades complejas, contradictorias, dinámicas y desafiantes originan que los ciudadanos requieran un nuevo 
contrato social con sus profesionales, que incide directamente en la formación profesional.

- Para responder a las necesidades sentidas de la población, son necesarios cambios fundamentales en los sistemas de atención de los servicios, en los modelos de práctica vigente, en la distribución geográfica de los recursos humanos y sobre todo en su formación.

- El bien más importante que posee el sistema de salud son sus recursos humanos, lo que se denomina "capital humano", y éste está en manos de los docentes que lo forman.

- El nuevo contrato social debe basarse en la voluntad de cumplir los compromisos de todos los involucrados en la relación sanitaria. Por una parte, los profesionales deben asegurar una prestación con elevados estándares de calidad. A su vez los ciudadanos, conscientes de sus derechos y deberes, deben pagar los impuestos para financiar el sistema y además deben cumplir con su propia responsabilidad, empoderándose del cuidado de su salud. Los responsables políticos deben garantizar el acceso equitativo a la atención sanitaria, deben gestionar la financiación del sistema y garantizar su calidad.

- La existencia de profesionales capacitados resulta una prioridad en el sistema de salud para la prestación del servicio a la población.

- Para lograr una elevada formación y de calidad, es fundamental el desarrollo de programas formativos y la interrelación de las instituciones de salud y las otras instituciones que intervienen en el proceso.

Con este marco se trabajó en la evaluación de las Residencias médicas de la provincia de Mendoza, Argentina. La evaluación de las Residencias de las profesiones de la salud fue compleja y requirió considerar las diferentes dimensiones: histórica, política, institucional, económica, social educativa y ética, entre otras.

Los resultados sirvieron como fundamento al anteproyecto de la Ley 7.857, promulgada en junio de 2008 en la provincia de Mendoza, Argentina. En la fundamentación de la ley se planteaba la necesidad de una propuesta innovadora que reafirmara el compromiso asumido en los ańos '70 por el Ministerio de Salud de la provincia. Además de señalar la capacitación de los profesionales como una prioridad dentro del propio sistema para la prestación de servicios de la salud a la población, se remarcaban algunos aspectos fundamentales para el desarrollo de los programas formativos, tales como la necesidad de la interrelación de las instituciones de salud con las instituciones de educación superior.

Dicha ley, que consta de 19 capítulos y 103 artículos, se creó para normalizar el funcionamiento del Sistema de Residencias del Ministerio de Salud y del Ministerio de Desarrollo Humano, Familia y Comunidad, de aplicación en el ámbito de la Administración Pública Provincial, organismos centralizados, descentralizados y autárquicos, y en la Obra Social de Empleados Públicos (OSEP) para profesionales de la salud. A través de su adherencia voluntaria se permite a los establecimientos asistenciales de los sectores de la seguridad social, el mutualismo, el privado o el estatal no provincial, con asiento en el territorio de la provincia de Mendoza, obtener el Reconocimiento Oficial.

Se puede afirmar que el nuevo marco normativo instaurado con esta ley se constituye en un hito histórico para la salud de los mendocinos. El cambio de paradigma que emana de la ley se esboza claramente cuando se afirma: "Las Residencias Profesionales son una estrategia educativa para la formación de sujetos sociales, democráticos y transformadores de la realidad. Se requiere para ello el compromiso de profesionales del mundo sanitario, que además de trabajar en campos interdisciplinarios sean capaces de actuar con otras disciplinas vinculadas directamente con la vida, la calidad de la misma y el ambiente". En este marco, son de destacar algunas de las novedades que propone la ley:

- Una red formada por profesionales formadores que interdisciplinariamente trabajan en los distintos niveles del sistema provincial de salud (se incorporan los aspectos positivos de la normativa reglamentaria de las Residencias médicas, pero se supera la hegemonía médi- 
ca incluyendo en su texto a los profesionales comprendidos en la ley $\mathrm{N}^{\circ} 7.759$ de la provincia).

- Tres pilares innovadores de la gestión educativa: las tecnologías de la información y comunicación (TICs), los valores y la evaluación como una estrategia de mejora e innovación de la calidad educativa.

- Formadores de distintas disciplinas que sean capaces de formar equipos de trabajos que puedan enseñar a aprender.

- Las Residencias de primer nivel suman al ciclo formativo prestacional un ciclo comunitario. Este tiene la finalidad de permitir al residente ejercer la especialidad en plenitud, en marcos y redes institucionales de salud, acrecentando destrezas, habilidades y actitudes. Se mantiene el nexo con el centro formador a través de una consulta semanal. El usuario se beneficia al recibir prestaciones donde hay carencias cuali y/o cuantitativas. Desde las políticas de RRHH se logra el fomento de asentamiento o inserción en lugares en los que se necesitan.

- La sociedad es reconocida con servicios por lo que aporta con medios y condiciones para que pueda haber formación.

La creación del Consejo Asesor Permanente de Residencias en el ámbito de la Dirección de Recursos Humanos del Ministerio de Salud, ha sido otro acierto de la Ley 7.857. Dec. 340/09(9). Está conformado por representantes de: Ministerio de Salud, Ministerio de Desarrollo Humano, familia y comunidad, gremio de los profesionales de la salud con representación mayoritaria (AMPROS), asociaciones profesionales, universidades públicas y privadas con carreras de grado cuyos egresados puedan acceder a residencias, sociedades científicas y representantes de los servicios formadores.

Sus objetivos son:

- Promover la difusión y conocimiento de la Ley 7.857 en las instituciones públicas (estatales y privadas) que expresen formalmente su intención de incorporarse como unidades formadoras, o que ya cuenten con Residencias.
- Supervisar la planificación de las obligaciones curriculares y la ejecución de la formación de posgrado de cada una de las Residencias con reconocimiento oficial.

La comisión trabajó con el marco de referencia señalado anteriormente.

La tarea fue ardua al principio, seguramente porque los mecanismos de cooperación institucionales son claramente insuficientes en nuestro medio, lo que acentúa barreras para el cambio, pero fue muy gratificante a medida que se diseñó y practicó un método de trabajo en que las acciones que se planificaban para mejorar las Residencias Profesionales lograban plasmar la opinión técnica, experiencias e intereses de todas las instituciones representadas. La implicación activa de los integrantes logró avanzar en forma consensuada en el diseño de instrumentos objetivos de evaluación de programas y de evaluación de centros que fueron difundidos a toda la comunidad. Fue evidente que la falta de colaboración entre expertos, profesionales y autoridades sanitarias, actúa como factor perjudicial para el sistema de salud, pero pueden contrarrestarse con voluntad política y con las normativas legales y técnicas y organizativas adecuadas.

También hay o deberá haber una Subcomisión Asesora por cada especialidad reconocida por los consejos deontológicos de las distintas profesiones. Cada Subcomisión por especialidad tiene una conformación semejante a la central, en el sentido de que cada uno de sus miembros representa a diferentes instituciones.

Son convocadas por la Comisión Permanente de Residencias - Consejo Asesor Directivo, para que asesore en las evaluaciones o toma de decisiones específicas de las distintas Residencias por especialidad y por profesión. Se rigen por un Reglamento Interno y deben contar con profesionales especialistas, miembros de las unidades formadoras, docentes universitarios con experiencia en Residencias (ex residentes, profesionales con experiencia en grupos o servicios formadores, de reconocido prestigio) y miembros o referentes de las sociedades científicas y de la Asociación Mendocina de Profesionales de la Salud. 
Las subcomisiones asesoras son responsables de poner en la práctica las normativas de la Comisión Permanente de Residencias, asesorando sobre pautas asistenciales, docentes, científicas y académicas, en lo referido a planificación, implementación, desarrollo y evaluación de la correspondiente Residencia. Además, deben realizar las visitas en terreno cuando la Comisión Permanente de Residencias - Consejo Asesor Directivo lo solicite, y controlar el cumplimiento de la Ley 7.857.

Uno de los primeros problemas que surgió al Consejo Asesor fue la del número de especialistas que es necesario formar. Para esto se tuvo en cuenta que hay dos elementos específicos: las necesidades de especialistas del sistema de salud de la provincia y la capacidad docente de cada uno de los centros formadores. Respecto del primer punto, está pendiente en nuestra provincia el análisis riguroso de la cantidad y ubicación geográfica de los profesionales de salud que se desempeñan actualmente y, además, la proyección de los requerimientos futuros. Esta tarea debiera ser llevada a cabo por la Comisión Provincial de Capacitación, Docencia e Investigación, tal como está previsto (Ley 7.759 y Ley 7.857), pero que todavía no se implementa.

La capacidad requiere de un análisis crítico a partir de los criterios de acreditación de cada una de las especialidades, tanto en aspectos cualitativos como cuantitativos. Los primeros se relacionan con el perfil curricular de los formadores, el tipo de tareas asistenciales que realiza y su actividad docente, de investigación y de relación con la comunidad. Los cuantitativos se valoran a partir del número de profesionales de la unidad formadora, la naturaleza de las tareas que realizan en dicha unidad y la aceptación de las autoridades del centro de permitir que parte de la carga horaria del prestador sea dedicada a la tarea docente. Le corresponderá a la Comisión Provincial de Capacitación, Docencia e Investigación analizar independientemente la necesidad de especialistas y la capacidad docente, para que ninguno tenga preponderancia sobre el otro. Sobre todo porque la capacidad docente no puede ser superada para disponer de un número mayor de especialistas. Tampoco el número de especialistas a formar debe basarse en las necesidades asistenciales de la unidad docente.
Otra de las tareas desarrolladas se refiere al Sistema de Acreditación y Reacreditación de Centros y Unidades Docentes. Se han establecido con claridad, por parte del Consejo Asesor Permanente, los criterios de acreditación y reacreditación de centros o unidades de formación y se han hecho públicos para lograr transparencia. En cada centro y en cada una de las Residencias deben instalarse sistemas de autoevaluación.

Está pendiente para la siguiente etapa el desarrollo de un sistema de acreditación para los formadores de la residencia, con el fin de garantizar la inserción adecuada de los residentes en cada uno de los servicios.

En lo que se refiere al contexto institucional, se ha difundido en distintas instancias de enseñanza-aprendizaje que, para lograr un perfil de excelencia de los residentes, el contexto institucional debe propiciar el desarrollo académico, científico, organizacional, profesional y ético.

Los objetivos de la Residencias deben ser explícitos y se deben corresponder con las actividades programadas, que deben ser congruentes con el perfil.

Los programas formativos, son una herramienta imprescindible para guiar el desarrollo de cada una de las actividades de la Residencias.

De manera general, entendemos a la evaluación de los programas como aquel conjunto de procesos sistemáticos de recogida y análisis de información fiable y válida para tomar decisiones sobre un programa educativo.

La actualización periódica y frecuente de los programas formativos de las diferentes Residencias es necesaria. Asimismo, las diferentes especialidades deben poseer una homogeneidad en estructura y contenidos.

La actualización y perfeccionamiento de los planes de formación debe efectuarse en función de estándares regionales e internacionales comparables, pero respetando los contextos.

También debe tenerse en cuenta para cada una de las especialidades la información que surge de procesos de evaluación y acreditación nacionales, 
regionales e internacionales (CONEAU, ARCUSur y RIACES)

Cada centro formador debe adjuntar el programa de residencia propuesto con los distintos elementos que la ley especifica, pero hay elementos que son esenciales: la misión institucional, los valores y principios, los actores y sus respectivos roles, el marco organizativo y el marco normativo.

La misión, los valores y principios establecidos en el marco estratégico son referentes esenciales de transmisión a los residentes, en tanto conforman la identidad y la razón de ser de cada institución.

Cada programa se debe organizar a partir del perfil que se desea alcanzar, lo que algunos denominan como meta curricular.

En su formulación se debe tener en cuenta que se corresponda con los criterios básicos de la especialidad, se contemplen campos completos del ejercicio profesional o del área disciplinar y se logre a través de las actividades institucionales programadas.

Para las residencias de primer nivel, la ley define cada una de las áreas que se deben programar: Asistencial, Gestión, Investigación, Educación para la Salud y TICs.

En los programas deben estar claramente definidas las competencias y los periodos en que se deben lograr las competencias genéricas y específicas. Deben también detallar el número mínimo de procedimientos que deben realizar los residentes, como garantía cuali-cuantitativa del proceso formativo y las competencias transversales para el conjunto de especialidades o subgrupos de especialidades afines. El cumplimiento de estas competencias transversales debiera estar garantizado por el Ministerio de Salud, en articulación con otras instituciones prestacionales o educativas, públicas o privadas. Este modelo se puede convertir en una excelente oportunidad para aprender a aprender juntos distintos profesionales y a su vez desarrollar capacidades para trabajar en equipos para responder a las demandas sociales con excelencia.

Los otros elementos del diseño curricular que se deben consignar son los contenidos, las estrategias de enseñanza aprendizaje y los recursos didácticos.

En términos estratégicos, los programas de todas las residencias deben guiarse, entre otros, por los Objetivos de Desarrollo del Milenio de las Naciones Unidas, la Agenda de Salud de las Américas 2008-2017 y las Metas Regionales de Recursos Humanos para la Salud 2008-2015.

La evaluación merece una consideración especial y debe contemplar tanto la planificación y el diagnóstico (al inicio), la aplicación —a la cual también podemos llamar de proceso o formativa-, como la evaluación de resultados, sumativa o integradora. En cada momento de la evaluación, los distintos actores involucrados pueden elegir diferentes elementos que constituyen distintos objetos de evaluación que son evaluados de diferentes modos.

¿Qué evaluamos? Las características del programa, los contenidos y las competencias deben ser evaluados antes de comenzar cada ańo de residencia. Durante el desarrollo se evalúa el proceso de aprendizaje de cada residente y su participación en las actividades programadas. Al finalizar el año se evalúa el rendimiento y desempeño de los residentes, así como la adecuación de los recursos educativos (materiales, bibliografía) y actividades para lograr el perfil deseado por cada uno de los residentes.

\section{Conclusión}

La complejidad y heterogeneidad del campo de la salud hace que lo veamos como un terreno que excede el abordaje desde una sola disciplina y requiere para su desenvolvimiento eficaz de la convergencia de diferentes discursos, prácticas y lecturas de la realidad.

Los cambios de paradigma han surgido como resultado de la reflexión crítica de las tendencias clásicas, los aportes de la sociología de las organizaciones, el análisis institucional, los nuevos conceptos en educación de adultos y la bioética. Desde este cambio de paradigma, todos los profesionales de la salud deben contribuir a alcanzar el mejor desempeño en el sistema de salud, teniendo 
en cuenta no sólo los problemas prioritarios, sino también la capacidad de anticiparse a las necesidades de dicha población y ser capaces de propiciar canales de participación y deliberación de todos los involucrados. Esto lleva a requerir nuevas competencias a los profesionales residentes $y$, a su vez, los formadores deben garantizar los mecanismos de acreditación de los procesos, así como el impacto que se logra en la salud.

Si se sostiene el carácter histórico-social del proceso salud-enfermedad-atención, la salud como derecho social y la necesidad de políticas activas para garantizarlo, se debe considerar a las Residencias como una parte importante de los recursos humanos y, por lo tanto, como actores fundamentales para transformar a la salud.

El actual marco legal propicia profundos cambios, tales como, la intersectorialidad, la organización y pertenencia a redes, la prioridad del primer nivel y la interdisciplina.

Se hace necesario concebir la cooperación como parte integrante de las misiones de las instituciones, que deberán crear herramientas o mecanismos convenientes para promover y regular dicha cooperación.

Referencias

1. Forest JJ, Altabach PG, (eds). International Handbook of Higher education, vol. 1. The Nederlands: Springer Publishers; 2006.

2. Knight J. Dudas y conflictos en torno a los programas dobles. En: Globalización e internacionalización de la educación superior. Revista de Universidad y Sociedad del Conocimiento 2011; 8(2). Disponible en http://www.redalyc.org/ articulo.oa?id=78018793011www.cres.2008. cartagena Consultado el 13/11/13.

3. Global Consensus for Social Accountability of Medical Schools Disponible en http://www.healthsocialaccountability.org Consultado el 13/11/13.

4. Organización Panamericana de la Salud. Agenda de Salud para las Américas 2008-2017. Bioética: Hacia la integración de la ética en el ámbito de la salud. Presentada por los ministros de salud de las Américas en ciudad de Panamá, en la XXXVII Asamblea General de la Organización de los Estados Americanos, junio de 2007. Washington DC: OPS; 2007. Disponible en: http://www.paho.org/Spanish/DD/PIN/Agenda_de_Salud.pdf. Consultado el 13/11/13.

5. Organización Panamericana de la Salud. Establecimiento del programa regional de bioética 37o Consejo Directivo de la OPS, 45a sesión del Comité Regional de la OMS para las Américas; del 27 de septiembre al 1 de octubre de 1993. Washington DC: OPS; 1993. Disponible en: http://bases.bireme.br/cgi-bin/wxislind.exe/iah/online/?IsisScript=iah/ iah.xis\&src=google\&base=LILACS\&lang=p\&nextAction=lnk\&exprSearch=371653\&indexSearch=ID Consultado el 13/11/13.

6. República Argentina. Ley 7.857. Boletín Oficial de Provincia de Mendoza, 27 de junio de 2008.

7. Beaglehole R, Bonita R, Horton R, et al. Public health in the new era: improving health through collective action. Lancet 2004; 363: 2084-2086.

8. Stahl T, Wismar M, Olilla E, (eds.) Health in all policies, prospects and potentials. Finland: Ministry of Social Affairs and Health; 2006

9. República Argentina. Ministerio de Salud. Decreto 340/09. Publicado el 30/03/09. 